\title{
1 Form follows function: Werkzeuge der internen Kommunikation
}

\author{
Frank Martin Hein
}

- Die Kommunikation im Forschungsverbund kann stark von den Erfahrungen von Fachgemeinschaften (Communities of Practice) profitieren.

- Interne Kommunikation ist mehr als ein Verlautbarungs-Instrument. Sie organisiert den Informationsaustausch, der maßgeblich zum Erfolg der Gruppe beiträgt.

- Die Arbeit und Sach-Kommunikation in Gruppen wird von der spezifischen Gruppendynamik der Beteiligten überlagert.

- Vertrauen zueinander zu erwerben, zu wahren und zu mehren, erfordert die permanente Aufmerksamkeit aller.

- Die Glaubwürdigkeit der Sender bei den Empfängern muss konsequent erarbeitet werden.

- Kommunikation im Forschungsverbund ist Mittel zum Zweck - kein Selbstzweck.

\subsection{Ausgangsbasis}

Interne Kommunikation ist heute mehr als ein behäbiges Verlautbarungsinstrument von Führungseliten. Ihre Koordinatoren tragen maßgeblich zur Zielerreichung der Organisation bei. Sie pflegen aktuelle Diskussionsforen jeder Art, verfassen Newsletter und entwickeln das ,Intranet' zur übergreifenden Wissensbank. Leicht zu bedienende elektronische Werkzeuge und 
knappe Budgets fördern dabei die Beteiligung interessierter Laien. In formellen wie informelleren Verbänden wie den ,Communities - also Fachgemeinschaften mit einem gemeinsamen Ziel - unterstützen sie nachhaltig den Zusammenhalt der Gruppe.

Auch wenn die spezifische Zusammenarbeit in der medizinischen Forschung mit räumlich getrennten oder sogar multinationalen Arbeitsgruppen, mit schwächer ausgeprägter formeller Hierarchie und stärker entwickelten fachlichen Rahmenbedingungen ganz besondere Anforderungen an die Kommunikation mit sich bringt: Die grundlegenden Mechanismen und globalen Trends greifen auch hier. Junge Wissenschaftler orientieren sich am außerakademischen Umfeld, bringen ihre privaten Medienpräferenzen, -Kenntnisse und -Erwartungen ein, nutzen den technischen Fortschritt und kommunizieren selbst. Die Kenntnis spezifischer Aspekte der Arbeit in Gruppen und professioneller, interner Kommunikation kann dabei ein wichtiger Erfolgsfaktor für alle Beteiligten werden.

\subsection{Kommunikation ist Mittel zum Zweck - kein Selbstzweck}

„Interne Kommunikation“ ist kein Selbstzweck. In jeder Organisation - egal ob Unternehmen, Verband oder Netzwerk - erwächst ihr Wert aus dem Beitrag, den sie zum Erfolg der Gruppe beisteuert, der bereits möglichst eindeutig für alle definiert ist oder als geteilte Zielvorstellung kommunikativ erarbeitet wird. Dabei sind Organisationen nur dann langfristig erfolgreich, wenn sie sich permanent an ihre Umwelt anpassen und sich mit den dadurch vorgegebenen Makrotrends arrangieren. Die alltäglichen Aufgaben - Transaktionen mit Dritten, die Projektplanung, der regelmäßige Austausch von Zwischenergebnissen vor einer Veröffentlichung usw. - möglichst reibungslos und effektiv zu bewältigen ist daher nicht mehr als eine notwendige Voraussetzung für das Überleben; hinreichend ist sie nicht, wenn das Team nicht nur für ein Kurzprojekt bestehen soll, wenn sich die Forschungslandschaft wandelt, wenn Fördergelder nach neuen Kriterien vergeben werden oder andere Arbeitsgruppen schneller Ergebnisse veröffentlicht haben. Für den amerikanischen Organisationspsychologen Edgar Schein sind die Prozesse der externen Adaptation und internen Integration jeder Gruppe von zentraler Bedeutung. Wie sie damit umgeht, charakterisiert ihre spezifische Kultur (s. Schein 2004). Die interne Kommunikation muss die zugehörigen Prozesse in bestmöglicher Weise unterstützen. Daher wird definiert:

Interne Kommunikation bezeichnet den organisierten Informationsaustausch zwischen den Angehörigen einer Organisation (eines Unternehmens, Verbandes etc.), um deren (dessen) Ziele zu erreichen. 
Wichtig ist, dass Kommunikation als zielgerichtet organisierter Prozess verstanden wird - und nicht als private oder zufällige Angelegenheit und dass sie den Zielen der Organisation dient - und nichts anderem. Dabei ist es hilfreich, die Angehörigen dieser Organisation klar zu definieren (Gruppenbildung) und deren Integration zu fördern. Und wichtig ist schließlich, dass ein Informationsaustausch stattfindet - ein Prozess, der einerseits in mindestens zwei Richtungen abläuft, und andererseits relevante Inhalte transportiert. Informationen beseitigen Unsicherheit beim Empfänger - Daten allein tun dies nicht. Nicht alles, was ein Absender für Information halten mag, wird auch vom Empfänger als relevant empfunden. Die Medien der internen Kommunikation stellen die Mittel, um die geschilderte Aufgabe zu erfüllen. In diesem Beitrag geht es daher um die folgende Frage:

Wer kommuniziert mit wem, warum, was, wie und mit welchem Ergebnis?

\subsection{Teil I: Wer, wie und mit wem - Definition und Integration der Gruppe}

Die Arbeit in Gruppen erfordert nicht nur eine technisch funktionierende Kommunikation der Mitglieder untereinander, diese wird automatisch von der spezifischen Gruppendynamik der Beteiligten überlagert. Zusätzlich unterliegt dieser Prozess dem kulturellen Einfluss der Organisationen, in die er eingebettet ist. Deren Eigenheiten können den Erfolg der Gruppenarbeit schmälern.

Wink (2007): „Ein spezielles Problem für Forscher innerhalb internationaler und interdisziplinärer Gruppen stellt die Zugehörigkeit zu unterschiedlichen ,Forschungskulturen` dar, d.h. die Hierarchiebildung, Kommunikationsform und Kommunikationsregeln sind zumindest zwischen asiatischen, europäischen und nordamerikanischen Gruppen, zum Teil aber auch zwischen einzelnen Nationen oder Instituten, unterschiedlich.“

Es lohnt sich, auf den gut dokumentierten Erfahrungen sog. ,Communities“ aufzubauen, die oft ähnliche Probleme zu bewältigen hatten - traditionell noch ohne elektronische Medien. Man unterscheidet drei Arten von Communities (Fachgemeinschaften), die sich in der Realität oft überlagern:

- Communities of Interest definieren sich über ein geteiltes Interesse an einem Thema;

- Communities of Purpose sind Zweckgemeinschaften meist kürzerer Lebensdauer, die eingerichtet werden, um spezifische Ziele zu erreichen; am wichtigsten aber sind hier

- Communities of Practice. Hildreth et al. 2000 definieren sie als „eine Gruppe von Professionals, die informativ miteinander verbunden sind 
durch ihre gemeinsame Beziehung zu einer Klasse von Problemen oder der Suche nach Lösungen und so ihrerseits einen Wissenskörper darstellen. "In diese Kategorie fallen die formelleren Forschungsverbünde in der Medizin und die Deutschen Zentren der Gesundheitsforschung.

Wenger (1998) definiert sie entlang der drei Dimensionen: gegenseitiges Engagement, gemeinsames Vorhaben (explizit als Mission oder Ziel artikuliert, aber ständig neu unter den Mitgliedern verhandelbar) und gemeinsames Repertoire (Wörter, Werkzeuge, Geschichten, Gesten, Symbole, Routinen, Konzepte, usw.). Neue Mitglieder lernen von den älteren in einem sehr umfassenden, sozialen Prozess. Dabei erfahren und vermitteln die Mitglieder Bedeutung. Sie tauschen sich aus über gemeinsame historische und soziale Erfahrungen und Perspektiven, die das gemeinsame Handeln bestimmen (,Practice'). In diesen Gemeinschaften (,Communities') sprechen die Mitglieder über die Unternehmungen, die sie verfolgen und in denen sie als kompetent erkannt werden. Während sie interagieren, formt sich ihre Identität und persönliche Geschichte im Kontext der Organisation.

Communities bilden sich normalerweise spontan rund um Persönlichkeiten oder gemeinsam erkannte Probleme. Die Mitglieder rekrutieren sich gegenseitig, zum Beispiel, um bestimmte Aufgaben zu lösen, die als fachlich wichtig erkannt werden. Die daraus folgende Kommunikation ist individuell, zumindest so lange die Community keine offizielle Funktion ausfüllt. Werden Communities offiziell eingesetzt oder sogar gefördert, handelt es sich eher um Projekt-Teams, formelle Arbeitsgruppen oder richtige Abteilungen - die Kommunikation ist dann automatisch institutionell, und es werden definierte Ergebnisse erwartet. Einerseits profitieren die Communities dadurch von offizieller Reputation und Ressourcen, andererseits fällt die motivierende Kraft des eigenen Engagements weg, die Leidenschaft für die Sache kann von hierarchischen Effekten überlagert oder entwertet werden.

Im Einzelfall kann es viel sinnvoller sein, eine inoffizielle Cruppe stillschweigend zu tolerieren, als ihr um jeden Preis einen offiziellen Status zu geben. Was zählt ist, dass die Mitglieder einen persönlichen, langfristigen Zweck für ihr Engagement erkennen. Kim (2000): „Beständige Communities haben ein beständiges, nachhaltiges Geschäftsmodell hinter sich“. Und das kann durchaus solider in der beruflichen Neugier und wiederkehrenden Jahreskonferenzen der Spezialisten zum Thema verankert sein, als in einem kleinen monatlichen Zusatzeinkommen. Das Spannungsverhältnis zwischen offizieller, geförderter Arbeit und inoffizieller, selbstmotivierter, kann im Kontext strenger Förderkriterien und angesichts der prekären Lebensverhältnissen vieler Nachwuchsforscher wohl nur durch weitsichtige Führungspersönlichkeiten aufgebrochen werden, die den Weg für praktische Kompromisse ebnen können.

Hildreth et al. (1998, 2000) haben sich ausführlich mit internationalen Communities of Practice beschäftigt und kamen in einer Feldstudie zum (nahe 
liegenden) Schluss, dass „die zugehörigen Menschen der wichtigste Faktor in einer Fachcommunity sind - deren Chemie untereinander und Bereitschaft, Dinge untereinander zu teilen und sich zu unterstützen“. Dabei verschiebe sich die Kommunikation von 1:1 hin zu Varianten der Kommunikation von 1:Vielen. Sei dieses Kriterium erfüllt, würden auch formelle Gruppen entsprechend arbeiten können. Was nicht funktionieren würde, sei bei nicht an einem Ort konzentrierten Mitgliedern „einfach die Eigenschaften von Faceto-face-Cruppen virtuell reproduzieren zu wollen“. Räumlich stark verteilte Communities können sich auflösen, wenn lokal viele weitere starke Bindungen bestehen, die Aufmerksamkeit für ihre Zwecke fordern.

Wenger et al. (2002) stellen sieben generelle Prinzipien heraus, wie Communities of Practice gefördert werden können:

1. zukunftsfähige Gestaltung, die künftige, dynamische Entwicklungen fördert;

2. ein offener Dialog zwischen interner und externer Perspektive;

3. Niveaus für verschieden intensive Teilnahme;

4. Raum für öffentliche und private Interaktion der Mitglieder;

5. Betonung der Wertschöpfung, des Nutzens der Teilnahme für ihre Mitglieder;

6. eine Kombination von Heimatgefühl und Anregung;

7. ein gemeinsamer Rhythmus, ein Tempo.

Viele dieser Aspekte lassen sich auf die verteilten Gemeinschaften übertragen, als solche ja oft auch wissenschaftliche Arbeitsgruppen organisiert sind. Zentraler Aspekt dabei ist wiederum, wie die Zusammenarbeit geregelt wird. Um beispielsweise die Stabilität von Online Communities ${ }^{1}$ zu fördern, kommt es laut Kim (1999) insbesondere darauf an, Erwartungen zu managen, insbesondere, was den Altruismus der Teilnehmer anbelangt. So wird geschätzt, dass 10\% der Mitglieder für 90\% der Aktivitäten von freien Online Communities verantwortlich sind. In der Fachliteratur spielen die Bemühungen, die übrigen 90\%, die „Lurkers“, einzubeziehen, eine große Rolle. Ihnen muss ein konkreter Wert geboten werden. Wer sich die Erfahrungen mit virtuellen Communities für größere Verbundvorhaben zunutze machen will, profitiert von einer kritischen Reflektion der folgenden Fragen im (virtuellen) Team:

- Welche Medien/Interaktionen führen am besten zu den Zielen der Gruppe?

- Welchen Wert hat die Community für ihre Mitglieder?

- Welchen Wert hat die Community für die Zielerreichung?

- Können die Mitglieder den Wert der Mitgliedschaft schnell erklären?

- Von welchen anderen Cruppen (intern wie extern) kann gelernt werden?

1 Rein physische Communities werden immer seltener. Siehe auch Fußnote 3 
- Werden die Erwartungen der Mitglieder hinreichend gemanagt?

- Wird für die Kommunikation untereinander geschaffener technischer Raum auch sozial gefüllt?

- Passt der Umgang im (virtuellen) Team zum Menschenbild der Organisation?

- Wie werden vereinbarte Regeln durchgesetzt?

- Wird der Umgang der Menschen miteinander gefördert - wie?

- Werden eine gemeinsame Geschichte, Sprache, Rituale gepflegt?

Virtuelle Teams zeichnen sich generell dadurch aus, dass in diesen eine Gruppe von Menschen unabhängig von Zeit, Raum und Organisation gemeinsam an einem Ziel oder einer Aufgabe arbeitet (s. dazu auch Lipnack 1995, 2000). Man will einerseits enge, etablierte Gruppen, in denen sich die Menschen blind vertrauen und austauschen, andererseits gibt es Situationen, in denen gerade bislang fremde Mitglieder der Organisation einbezogen werden müssen, um Erfolg zu haben. Es kann die Effektivität stark erhöhen, an Kernprozessen als eingespieltes, homogenes Team zu arbeiten, in dem die typischen Phasen der Gruppenbildung und kultureller Lernprozesse bereits durchlaufen wurden. Um neue, alternative Ideen zu finden, sind homogene Gruppen aber nicht unbedingt der beste Weg (Diehl u. Ziegler, S. gof.), diskutieren diese doch „,vor allem jene Informationen, die bereits allen bekannt sind.“ Die Autoren führen dafür drei Gründe an. So sei erstens statistisch offensichtlich, „dass eine Information, die allen Mitgliedern einer Gruppe bekannt ist, mit höherer Wahrscheinlichkeit in einer Gruppendiskussion eingebracht wird als eine Information, die nur einem einzigen Gruppenmitglied bekannt ist. Geteilte Informationen werden gegenüber nicht geteilten Information mit überproportional höherer Wahrscheinlichkeit genannt und diskutiert.“ Zweitens stimuliere eine Diskussion unter Gleichen natürlich eher solche Assoziationen, die im Gedächtnis aller Gruppenmitglieder vorhanden seien, als solche, die nur bei einem einzigen Gruppenmitglied vorhanden sind. Drittens würde ein offener Austausch in der Gruppendiskussion auch die Tendenz zu konformen Verhalten fördern. Man bezeichnet es als „Groupthink“, wenn das Streben nach Einmütigkeit in einer kohäsiven Gruppe die Fähigkeit zu einer rationalen Entscheidung nachhaltig verdrängt (vgl. Janis 1973). Die Folge ist, dass selbst bereits vorliegende Information „nur unzureichend oder verzerrt diskutiert werden, abweichende Meinungen und Handlungsalternativen keine Berücksichtigung finden und die Diskussion mit Personen außerhalb der Gruppe vermieden wird. "Surowiecki (2004) hält es deswegen für wesentlich riskanter, eine kleine, homogene (selbst hoch qualifizierte) Gruppe wichtige Entscheidungen fällen zu lassen als eine größere, diversifizierte².

2 Vgl. dazu Schein 2004, der es die Ausbildung von Unternehmenskultur gerade davon abhängig macht, als Gruppe gemeinsam Probleme zu lösen. 
Ein viertes Problem homogener - oder danach strebender - Gruppen ist der soziale Vergleich. Dieser kommt dann zum Zuge, wenn sich eine Aussage nicht an der äußeren Realität, sondern nur durch den Vergleich mit der Meinung Dritter überprüfen lässt. Eine Aussage gilt dann als richtig, wenn möglichst viele andere sie teilen (Asch 1946). Diehl und Ziegler (2000): „Diese Konsensheuristik mag zwar in vielen Fällen angemessen sein, sie führt aber auch dazu, dass Informationsaustausch vermieden wird, um einen bestehenden Konsens nicht zu gefährden. Ein auf mangelhaften Informationsaustausch beruhender Konsens ist jedoch häufig Nonsens." Und Surowiecki betont, dass Entscheidungen weniger gut werden, sobald Individuen aufhören, sich auf ihr eigenes Wissen zu verlassen und stattdessen Entscheidungen darauf basieren, was sie glauben, was andere Leute wissen. Eines der größten Probleme von Gruppen sei es, Menschen dazu zu bringen, viel weniger auf das zu hören, was andere sagen.

\subsection{Die Arbeit in großen Forschungsverbünden - gelebte Kommunikation}

Das Team gut starten. Idealerweise steht am Anfang ein physisches Meeting. Internationale Teams legen zu Beginn eine gemeinsame Sprache fest. Manche der von Majchrzak et al. (2004) befragten Leiter räumlich verteilter, virtueller Teams ${ }^{3}$ verwandten explizit Zeit darauf, Gemeinsamkeiten unter ihren Teammitgliedern zu finden und zur Sprache zu bringen. Für viele Teams war es nützlich, die Persönlichkeiten ihrer Mitglieder zu Beginn zu diagnostizieren und sich später explizit auf die gefundenen persönlichen Eigenschaften zu beziehen. Gute Teamführer erklären die Aufgabe ausführlich und bereiten Mitglieder auf ihre Rollen vor. Wenn möglich, wird eine Teamcharta verfasst. Teamprozesse, Unteraufgaben und Teilschritte werden gemeinsam entwickelt. Es wird eine Etikette für das Projekt etabliert. In dieser Phase entsteht erstmals so etwas wie Stallgeruch und Wir-Gefühl. Man betont den anhaltenden Charakter der Zusammenarbeit - es lohnt sich also für die Teilnehmer, sich als Person und in deren Beziehungen zu investieren. Eine solche eher umfangreiche Anlaufphase zahlte sich in jedem Fall später aus.

Teamcharta abfassen. Eine Team-Charta enthält die Vision, Mission und Regeln für den Umgang miteinander. Sie formuliert Aufgabe und Ziel des Teams sowie die Kriterien, an Hand derer Erfolg bestimmt wird. Sie listet Kontaktdetails innerhalb der Gruppe und die relevanter Dritter auf. Dazu kommt die Etikette bzw. ein Verhaltenskodex (Ansprechen von Themen, Datenpflege,

3 Angesichts der flächendeckenden Verbreitung elektronischer Endgeräte, Medien und Datenbanken ist die Existenz rein physischer Teams im akademischen Umfeld kaum noch vorstellbar. Schon der Brückenschlag zwischen zwei Laboren, umso mehr zwischen mehreren Etagen, Gebäuden und Standorten macht größere Teams zumindest zeitweise virtuell. Diverse Studien belegen den sinkenden persönlichen Austausch durch wachsende räumliche Distanz. Gezielte Maßnahmen der internen Kommunikation helfen, diese zu überbrücken. 
Austausch, Stil, Ansprechen persönlicher Themen, Lösen von Konflikten und Problemen, Einhalten von Versprechen, Datenpflege). Gibt es für die Gruppe permanent wichtige, gemeinsame Dokumente, werden diese integriert. Die Charta kann um Profile und Rollen der Teammitglieder ergänzt werden. Je offener sich diese darin vorstellen, desto besser. All das wird online ständig prominent verfügbar gehalten.

Gruppenprozesse kennen und berücksichtigen. Klassische und virtuelle Teams haben stets soziale und Aufgaben-bezogene Prozesse zu bewältigen. Bei der klassischen Gruppe werden die sozialen Aspekte schneller und offener abgehandelt. Sie zeigen sich auch durch Körpersprache; Probleme sind relativ leicht erkennbar. Bei den virtuellen Teams gibt es eine versteckte, verzögerte Dynamik (Rollen, Kompetenzen, Sympathie), die oft über die Technologien ausgetragen wird (welche Medien, wie genutzt); Probleme werden so verdeckt. Die Aufgaben-bezogenen Aspekte stehen bei klassischen Teams zunächst im Hintergrund. Bei virtuellen Teams steht die Aufgabe zunächst vermeintlich im Vordergrund; konstruktive Arbeit beginnt oft bilateral, die produktive Phase ist dann multilateral, technische Hilfsmittel werden gemeinsam genutzt; Sacharbeit führt zu Teaming. Wenn es Probleme mit virtuellen Teams gibt, sind diese eher sozialer Art. Technologie ist nur ein Mittel zum Zweck.

Vertrauen untereinander fördern. Die Mitglieder virtueller Teams können sich nur selten in die Augen schauen und als soziale Wesen über das Projekt hinaus erleben. Vertrauen zu einander zu erwerben, es zu wahren und zu mehren, erfordert deswegen die permanente Aufmerksamkeit aller. Es kann sich lohnen, diesen Aspekt im virtuellen Team selbst zu adressieren und zu diskutieren, sowie sich selbst dabei ständig zu überprüfen. Dazu gehören Aspekte wie

- Kompetenz: d.h. die Fähigkeit, sich selbst zu steuern und korrekt einzuschätzen, den Wissenstand im eigenen Verantwortungsbereich aktuell zu halten, die Fachgebiete der anderen Teammitglieder zu respektieren, Anregungen und Kritik Dritter aufzunehmen, Selbstdisziplin, Aktives zuhören, Frustrationstoleranz und Medienkompetenz.

- Mitarbeit: Motivation, Versprechen geben und zuverlässig halten, Aufgaben übernehmen und sachgemäß durchführen, Ideen einbringen und neuen Teammitgliedern helfen, Informationen teilen, rechtzeitig alle bei Veränderungen warnen.

- Integrität: Keine Kritik an Personen vor Dritten äußern, keine Widersprüche generieren (Worte/Taten, Werte/Aktionen, Menschenbild/Sprache usw.), dem kategorischen Imperativ folgen, konsistent in alle Richtungen argumentieren, eigene Handlungen und Positionen erklären und nachvollziehbar machen, Versprechen einhalten.

- Fairness: Die Arbeit im Team angemessen verteilen, an Standardprozessen und -Normen messen, ohne zu bevorzugen oder zu benachteiligen, alle Mitarbeiter nach den gleichen, nachvollziehbaren Kriterien belohnen/befördern, Individuen respektieren. 
Das Team zusammenhalten. Auch diese Aufgabe zerfällt in eine sachliche und eine soziale Komponente. Sozial geht es darum, das bereits vorhandene Wir-Gefühl durch den Bezug auf gemeinsame Ziele, Erlebnisse, Erfolge und Feinde permanent zu stärken und das gegenseitige Vertrauen zu fördern. In der Sache geht es darum, die Priorität der Aufgabe auch über die Distanz zu erhalten und die einzelnen Beiträge zu strukturieren. Kontakte sollten häufiger stattfinden als in physischen Gruppen. Arbeitsfortschritte werden genau verfolgt, auch kleine Erfolge und vorbildliches Verhalten gefeiert. Teamleiter können temporäre Paare von Teammitgliedern bilden, um Unteraufgaben zu lösen und den sozialen Zusammenhalt zu fördern. Schüchterne sind zu coachen (aber auch bei Misserfolg zu ignorieren). Ein guter Teamleiter schafft immer wieder Gelegenheiten, in denen die Teilnehmer direkten Kontakt haben und ihre soziale Beziehungen festigen können und sorgt dafür, dass der gemeinsam zurückgelegte Weg gut zugänglich für alle dokumentiert wird.

Strukturierter kommunizieren. Fragen, Antworten, Erwartungen, Verabredungen und Vereinbarungen sollten viel deutlicher als solche gekennzeichnet werden als üblich. Bei Konversationen sollte man sich auf seine Vorredner namentlich - auch durch Zitate - beziehen. Gerade die Teamleitung sollte virtuell ständig präsent und ansprechbar sein. Die Wahrscheinlichkeit von Attributionsfehlern sollte minimiert werden. Deswegen ist es wichtig, dass Verteilerlisten vollständig sind und der Erhalt von Botschaften bestätigt wird. Diese Rückkommunikation sollte schnell erfolgen.

\section{Beispiel Telefonkonferenzen}

Telefonkonferenzen sollten zu festen Zeiten und pünktlich stattfinden und von wechselnden Orten orchestriert werden (Aufbrechen lokaler Koalitionen). Die Teilnahme für jedes Teammitglied ist verbindlich, jedes Teammitglied entschuldigt sich persönlich, wenn es verhindert ist. Die Agenda wird vor den Meetings verschickt, diese werden nach einem festen Schema protokolliert. Alle Mitglieder der Gruppe sollten regelmäßig in die Konferenz einbezogen, Sprecher nicht unterbrochen werden. Soziale Aspekte haben ihren festen Platz zu Beginn, die Zusammenfassung von verbindlichen Aufgaben am Ende. Gelobt wird in der Konferenz oft und vor allen, kritisiert möglichst nur bilateral und separat.

\subsection{Teil II: Was interne Kommunikation leisten kann und was nicht}

Der Psychologe McClelland hat bereits 1995 Verantwortungsbewusstsein, Klarheit der Organisation und Teamgeist - das „richtige“ Klima - als ausschlaggebend für ein effektives Management ermittelt. Etliche Aspekte davon - in erster Linie Führungsfragen - wurden in den vorigen Abschnitten beleuchtet. Wie viel entscheidet sich nun durch die Arbeit der Netzwerkkoordinatoren, die mit der internen Kommunikation betraut werden? 
„Engagierte“ Mitarbeiter sind ein populäres Ziel, die positive Relation von ,Engagement ' zu ,Effektivität‘ und ,Produktivität' der Mitarbeiter wird dabei unterstellt. Das setzt einerseits voraus, Menschen und Aufgaben passend zuzuordnen, andererseits den Menschen eine Arbeitsumgebung zu schaffen, die ihnen die optimale Aufgabenerfüllung erlaubt. Angemessene Werkzeuge, Mittel, Prozesse und Informationen gehören dazu (vgl. Buckingham u. Coffmann 2002). Interne Kommunikation kann viel dazu beitragen, die zugehörigen Rahmenbedingungen, Anforderungen und Bedürfnisse zu klären und machbare Lösungswege herauszuarbeiten.

Interne Kommunikation gliedert sich dabei in einen Teil offiziell erstellter, somit institutioneller Kommunikation und einen Teil technisch geförderter, aber nicht inhaltlich gestalteter individueller Kommunikation durch die Mitarbeiter selbst. Je nach Zustand der Organisation und dem Verhalten der beteiligten Führungskräfte sind die Grenzen zwischen beiden fließend (vgl. Hein 2007).

Die institutionelle Kommunikation hat nicht nur die Aufgabe, reguläre Transaktionen zu unterstützen und die dafür notwendigen Informationen verfügbar zu machen. Noch wichtiger ist es, die Organisation in ihrer permanenten Anpassung an die sich wandelnde Umwelt zu unterstützen und potenzielle Lücken zwischen erforderlichen und erfolgten Handlungen systematisch reduzieren zu helfen. Dazu gehört es beispielsweise, externe Informationen über andere Arbeitsgruppen und deren Erkenntnisse bewusst und frühzeitig in das Team hineinzuholen. Institutionelle Kommunikation wird von den Mitarbeitern danach beurteilt, wie umfassend sie individuell wahrgenommene Fragen aufgreift und beantwortet - oder diese erst gar nicht aufkommen lässt. Tut sie all dies nicht, wird sie ignoriert. In Phasen des Wandels bleibt die offizielle, institutionelle Kommunikation immer wieder hinter den Erwartungen der Menschen zurück. Als Folge werden die von den Menschen erlebten Inkonsistenzen individuell diskutiert.

Individuelle Kommunikation folgt daraus, dass die Mitarbeiter die (betriebliche) Realität anders wahrnehmen, als sie offiziell dargestellt wird. Es wird institutionell anders argumentiert und gehandelt, als die Menschen es individuell erwarten. Folglich wird die Situation als widersprüchlich erlebt. Wenn die interne Integration und die Anpassung an die externe Umwelt nicht harmonieren, dann suchen die Mitarbeiter im individuellen Austausch nach Erklärungen und einer Lösung - beispielsweise, wenn die Experimente des eigenen Teams stark von der aktuellen wissenschaftlichen Diskussion abweichen oder Mittel gestrichen werden, ohne dass das nachvollziehbar begründet wird (s. Tab 1).

Je loser die Mitarbeiter sich ihren Chefs verbunden fühlen, je weiter die psychologische und physikalische Entfernung der Menschen voneinander ist und je geringer ihr Vertrauen in dauerhafte und belastbare organisatorische Strukturen, desto wählerischer werden sie sein - bei den Medien, wem sie 
Tab. 1 Der wahrgenommene Umfang interner Integration und externer Anpassung zeigt sich in der institutionellen und individuellen Kommunikation.

\begin{tabular}{llcl} 
& & \multicolumn{2}{c}{ Interne Integration } \\
Wahrnehmung: & hinreichend & unzulänglich \\
\cline { 2 - 3 } Externe Anpassung & hinreichend & institutionell & $\begin{array}{c}\text { institutionell } \\
+ \text { individuell }\end{array}$ \\
\cline { 2 - 3 } & unzulänglich & $\begin{array}{l}\text { institutionell } \\
+ \text { individuell }\end{array}$ & individuell \\
\hline
\end{tabular}

zuhören, was sie glauben und wem sie selbst die Wahrheit sagen. So entscheidet am Ende die Konsistenz aller von den Menschen erlebten Maßnahmen in Taten, Worten und Bildern mehr über den Erfolg der internen Kommunikation als der angebotene Medienmix. Was nicht bedeutet, dass man deren Auswahl dem Zufall überlassen sollte.

\subsection{Systematische Medienwahl folgt wenigen einfachen Prinzipien}

Organisationen können meist in grober Annäherung als Pyramide dargestellt werden. Es gibt ein Top-Management, Direktoren, Manager, sowie Fachkräfte in Büros, Werkstätten und im Außendienst. Je nach Struktur und Größe gibt es zudem Divisionen, Abteilungen und Länderorganisation. In Communities ist die Struktur meist weniger klar vertikal gegliedert und entspricht eher einem multipolaren Netzwerk. Forschungsverbünde als Organisationen stehen irgendwo dazwischen. Die Medienwahl folgt in jedem Fall dem Prinzip, einen vollständigen Kommunikationsfluss in alle Richtungen zu ermöglichen. Daraus folgen ein paar simple Schritte:

Zugangsmöglichkeiten prüfen. Nicht alle Mitarbeiter haben einen PC, gar mit Videokonferenzfunktion oder ein Smartphone. Manche sind viel unterwegs, andere haben während der Arbeitszeit keine Gelegenheit zu lesen usw. Es gilt daher, sich möglichst vor Ort über die Arbeitsbedingungen zu informieren und für alle passende, lebensnahe Lösungen zu finden: Ein Krankenpfleger setzt sich nicht unbedingt nach der physisch harten Arbeit noch an den PC, vielleicht aber wird auf dem Weg nach Hause das Smartphone konsultiert. Intranets mit integrierten Blogs können effektive Lösungen darstellen, solange sowohl Zeit gegeben wird, sie zu lesen wie sie mit Leben zu füllen. Für zeitlich besonders stark beanspruchte Klinik- und Abteilungsleiter - denen eigentlich eine Vielzahl privilegierter Quellen zur Verfügung steht - müssen oft knappe, maßgeschneiderte Formen gefunden werden.

Verbreitungszeit bedenken. Fast immer gibt es in einer Organisation Themen, die sehr rasch dem Team zur Kenntnis gebracht werden müssen, und solche, deren Vermittlung Zeit braucht und verträgt. Beides erfordert dedizierte Kanä- 
le. Zwangsläufig lassen sich gerade in größeren Organisationen Mitarbeiter nur nach Vorankündigung auch nur annähernd vollständig versammeln schon eher gelingen vielleicht Telefonkonferenzen, noch einfacher ist das Versenden von E-Mails oder SMS-Botschaften an alle. Poster hingegen haben eine längere Vorlaufzeit in der Produktion - aber bleiben dafür meist Jahre an den Wänden. Gedruckte Hauszeitschriften (Print) liegen zeitlich dazwischen.

Medieneigenschaften kennen und berücksichtigen. Die Rand Corporation (Wainfan $u$. Davis 2004) hat verschiedene Medien analysiert und verglichen. Die zentrale Frage dabei war, wie nahe der Übertragungsbreite einer klassischen menschlichen Interaktion (Face to face) damit gekommen wird und als wie gleichzeitig die Situation erlebt wird; „Print“ würde bei dieser Darstellung naturgemäß sehr niedrig liegen (s. Abb. 1). Je vollständiger man sich dem Austausch von Angesicht zu Angesicht annähert, desto geringer ist das Risiko von Missverständnissen. Kommunikation ganz ohne nonverbale Zeichen und Symbole, gerade über Kulturgrenzen hinweg, gleicht durch die damit verbundene Kanalreduktion einem Flug im Nebel - sie kann im besten Fall gelingen, man muss aber nicht ans Ziel kommen ...

Jedes Medium hat seine subjektive ,Anmutung“ - und objektiv belegbare Eigenschaften. Die Bandbreite elektronischer Medien ist effektiv kleiner als die eines Gespräches, die einer Instant-Message im Vergleich quasi atomar, die eines Videos liegt irgendwo dazwischen - je nachdem, wie intelligent es gemacht ist. Jedes Medium hat über die physikalischen Eigenschaften hinaus auch kulturelle. Es hat sich etabliert als schnell oder langsam, demokratisch oder autoritär, schwarz-weiß oder farbig, ehrlich oder demagogisch

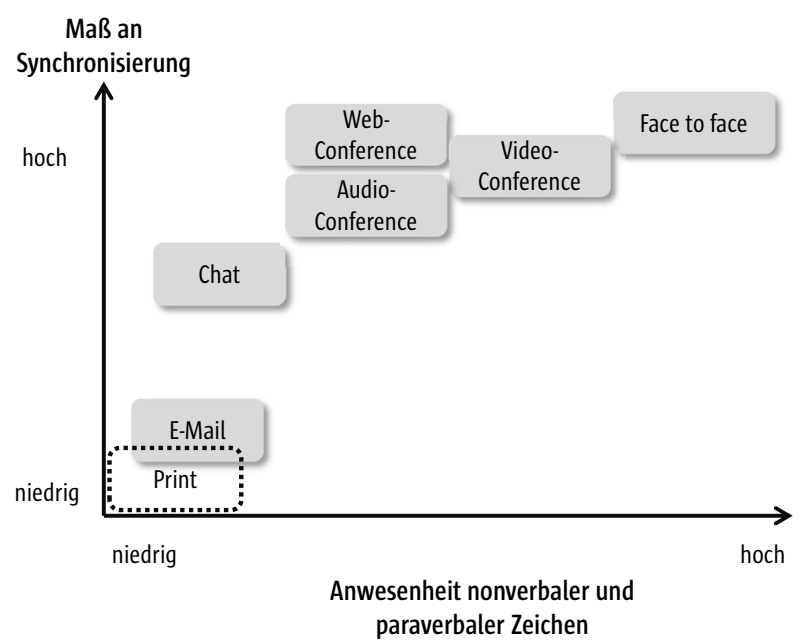

Abb.1 Kommunikation wird durch non- und paraverbale Elemente stärker synchronisiert (mod. nach Wainfan u. Davis 2004). 
im Ton. Je genauer die interne Nutzung den extern üblichen Funktionen der gleichen Medien entspricht, desto widerspruchsfreier fügen sie sich in die allgemeine Rezeptionserfahrung der Menschen ein. Interne Verschiebungen brauchen Zeit, völlige Abweichungen (z.B. eine undemokratische Nutzung sozialer Medien) schaffen Widersprüche in der Form, die von den Inhalten ablenken.

Einfache Basisstruktur festlegen. Es hat sich bewährt, eher weniger Kanäle nach klaren Prozessen mit abgestimmten Redaktionsplänen, Formaten und festgelegten Prozessen zu bespielen, als viele und stets neue Werkzeuge einzuführen, die sich im Endeffekt nicht aktuell, relevant und übersichtlich - also nutzerfreundlich - pflegen lassen. Crundlegend ist die Überlegung, was von Angesicht zu Angesicht, was gedruckt und was elektronisch kommuniziert werden sollte, und wer die jeweiligen Medien verantwortet. Ein Beispiel findet sich in Tabelle 2.

Die hier stellvertretend gezeigte, stark vereinfachte Struktur spiegelt eine eher hierarchisch geprägte Organisation mit mehreren Leitungsebenen. Größere physische Treffen haben jeweils einen Verantwortlichen, die Verwendung der elektronischen Medien ist nach der Wertigkeit von Nachrichten (Top-News versus Tagesgeschäft und Wissensablage im Wiki) gestaffelt, Druckmedien werden sparsam eingesetzt. Diagonale Wege können durch offene, elektronische Diskussionsplattformen oder formlose Feierabendmeetings realisiert werden, eine offene Diskussion kann durch Blogbeiträge und Foren alle Richtungen abdecken. Telefon- oder Videokonferenzen können in virtuellen Teams z.T. die physischen Meetings ersetzen, Beiträge im Internet und anderen externen Medien (hier nicht aufgeführt) wirken wiederum oft auch sehr effektiv in das Forschungsnetzwerk hinein.

Allen Mitarbeitern eine Plattform zur Artikulation ihrer Meinung zu geben kann schon deswegen sinnvoll sein, um die Diskussion offener Themen zunächst innerhalb des Forschungsverbundes zu halten und um mehr von dem mitzubekommen, was die Menschen wirklich bewegt. Je mehr sie auf den explizit dafür geschaffenen Einrichtungen offen fragen und diskutieren, desto mehr liegt ihnen ,ihre“ Organisation am Herzen. Ausufernde Diskussionen außerhalb sind ein Warnsignal, dass die Menschen sich nicht genügend auf offiziellen Wege beteiligen können.

Sprache beachten: Kommunikation ist nicht das, was man sagt, sondern das, was beim Empfänger ankommt. Sprache transportiert Kultur in konzentrierter Form. Sie kann Werte vermitteln oder ignorieren. Wie wir uns ausdrücken zeigt, wie achtungsvoll und aufmerksam wir mit unseren Konversationspartnern umgehen. Sagen wir die Wahrheit? Sagen wir sie deutlich? Wollen wir unseren Gesprächspartner Nutzen bieten, Überlegenheit zeigen oder Dinge verschleiern? Weisen wir an, stellen wir Fragen oder unterstellen wir Vorwissen oder Nähe? Gerade Computer-vermittelte Kommunikation ist zu erheblichen Teilen schriftliche Kommunikation, deren Qualität zugrunde 


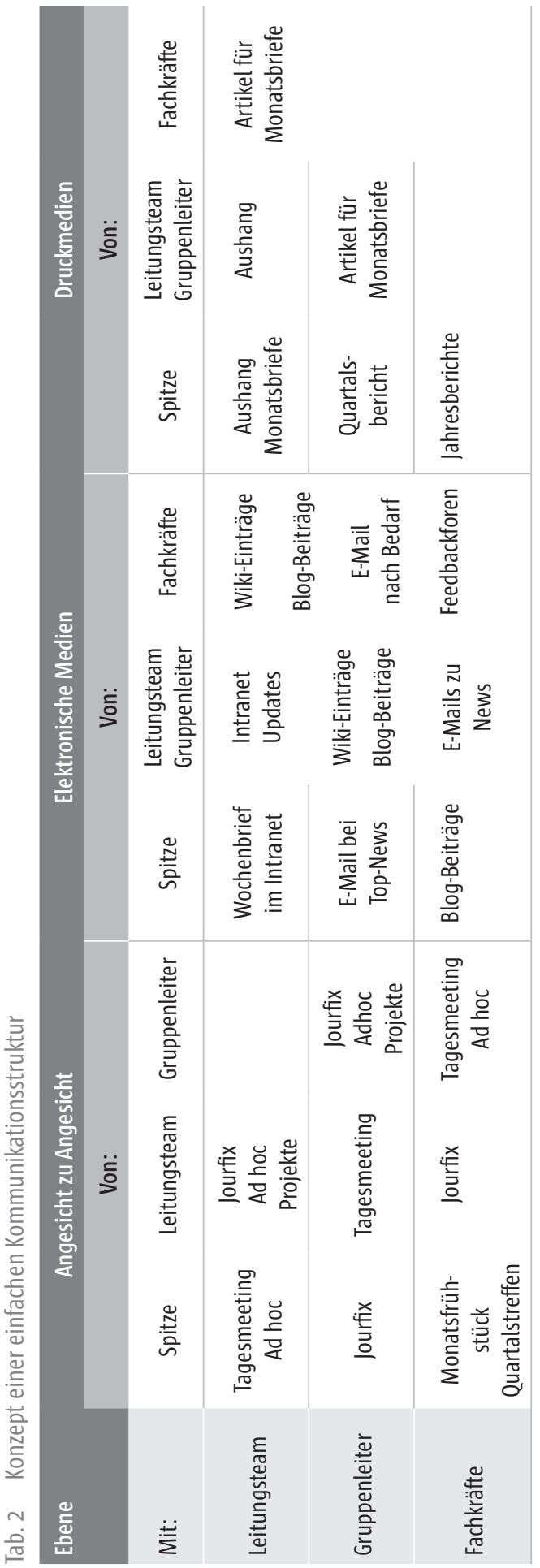


liegende Einstellungen nicht nur schnell deutlich macht, sondern auch langfristig dokumentiert.

Die Autoren der internen Kommunikation in Kliniken und Forschungszentren müssen sich darüber im Klaren sein, in welchem Umfang sie auf die Bedürfnisse ihrer Leser eingehen oder die Wünsche der Leitung transportieren. Wer dazwischen nicht vermitteln kann, hat es schwer. Werden werbliche Formulierungen verwendet - oder wird „Klartext“ gesprochen? Dürfen auch Führungskräfte formulieren „wie ihnen der Schnabel gewachsen ist“ - oder wird jeder Satz poliert? Nicht alles, was authentisch ist, ist gut. Nicht alles Gute ist authentisch. Poliert kann auch bedeuten: unglaubwürdig. Schreiben Laien, ist das zwar authentisch, aber genügt es auch einfachsten Kriterien an Verständlichkeit? Kommunikation muss stets zwischen divergierenden Motiven balancieren, was mit guter Vorbereitung besser gelingt. Hat die Organisation ein klar formuliertes, aktuelles Leitbild, einen Claim oder eine Charta sowie die drei bis fünf wichtigsten Botschaften für das Jahr verfasst, so lassen sich fast alle anderen sprachlichen Äußerungen daraus ableiten: Neue Botschaften spezifizieren die alten, aber ersetzen sie nicht. Aktuelle Vorhaben illustrieren die Pläne, aber machen kein „neues Fass auf“. Neue Texte beziehen sich auf bereits gemachte Aussagen, profitieren von der Änderung, der Wiederholung, der Iteration eines Themas, aber führen keine grundsätzlich neuen Gedanken ein. Es sei denn, etwas grundsätzlich Neues und Ungeplantes passiert und verlangt nach Deutung.

So vielfältig wie die verfügbaren Medien sind auch die sprachlichen Mittel, die ihnen dienen können. Der bereits erwähnte Nutzeneffekt für den Rezipienten gilt in elektronischen Medien in extremem Maße: Der Rezipient ist nicht durch das Protokoll einer physischen Begegnung gebunden, kann jederzeit die Lektüre abbrechen oder auf andere Medien ausweichen. Vom ,Aktiven Zuhören' abgeleitet und besonders zu bedenken sind daher die folgenden Fragen:

- Gibt es eine klare und eindeutige Bedeutung der Botschaft, wie lautet sie?

- Ist die Botschaft für die Zielgruppe interessant, nützlich und relevant?

- Werden erwartete Botschaften vorenthalten - wie wird das begründet?

- Befriedigt die Botschaft primär Interessen von Absender oder Empfänger?

- Wird der Absender so positioniert, wie er wahrgenommen wird/werden soll?

- Bewahrt die Botschaft Respekt und Achtung vor den Empfängern?

- Wird klar, welche Handlungen vom Empfänger erwartet werden?

- Regt die Botschaft zum eigenen Nachdenken oder Handeln an?

- Cibt die Botschaft Anweisungen an Einzelne, oder die Gruppe?

- Impliziert die Botschaft bestimmte Standpunkte oder Betrachtungsweisen?

- Respektiert die Botschaft verschiedene Perspektiven zu ungelösten Fragen?

- Adressiert die Botschaft alle Mitglieder einer Gruppe in gerechter Weise? 
- Stellt die Botschaft Absender oder Empfänger in eine besondere Beziehung zur Dritten (Personen, Abteilungen, Organisationen)?

- Kann die Botschaft dazu beitragen, Beziehungen zu bilden oder stärken?

- Passt die Botschaft zu existierenden Regelungen und Anweisungen?

- Kann die Botschaft jederzeit/vor jedermann ohne Probleme zitiert werden?

- Reflektiert die Botschaft kulturelle Werte, oder verletzt sie diese?

- Braucht der Empfänger (kulturelles) Vorwissen, um die Inhalte zu verstehen?

- Netiquette: passt der Ton der Botschaft zum Stil des Hauses?

Praxistipp: Viele Organisationen definieren ,Schreibregeln', die für alle Angehörige und deren interne Texte als Richtschnur gelten.

Kultur reflektieren. Wer sich praktisch mit interner Kommunikation befasst, vielleicht sogar beratend oder in leitender Funktion tätig sein will, hört oft Sätze wie: „Wir brauchen eine Erfolgskultur!“, „Ändern Sie bitte unsere Unternehmenskultur!“ oder gar „Motivieren Sie unsere Mitarbeiter!“. Das sind Forderungen, die ohne die aktive Hilfe aller Führungskräfte nicht erfüllbar sind. Die Psychologin Sonja Sackmann hat die umfangreiche Literatur zum Thema Unternehmenskultur systematisch ausgewertet und gezielt Dimensionen identifiziert, in denen ein Zusammenhang zwischen der Unternehmenskultur und dem Unternehmenserfolg unmittelbar nachgewiesen wurde (vgl. Sackmann 2006). Auch wenn keine dieser Dimensionen von den Mitarbeitern, die für „Interne Kommunikation“ zuständig sind, komplett verantwortet wird, so können sie doch einen erheblichen Teil zum Erfolg beitragen, weil sie die meisten Medien stellen, über die kulturelle Prozesse ablaufen. Und wo die Mitarbeiter der Kommunikationsabteilung dabei an ihre Grenzen stoßen - weil sie vielleicht nicht in jedem Meeting der Führungskräfte mit ihren Teams sitzen, können sie Beispiele und Best Practices zu diesen ,Kultur'-Dimensionen bieten:

- klare und kommunizierte Identität

- strategische (Ziel-)Orientierung

- Kundenorientierung

- Lern- und Anpassungsfähigkeit

- Innovationsfähigkeit

- Nutzen der Mitarbeiterpotenziale

- partnerschaftliche und kulturkonforme Führung - offene Kommunikation

- Leistungsorientierung/Leistungsbereitschaft und -fähigkeit

- balancierte Stakeholder-Orientierung

- strategische Passung

- Konsistenz zwischen normativem Anspruch und gelebtem Verhalten 
Vom Empfänger her planen. Gute interne Kommunikation kostet Geld. Sie ist eine Investition, deren Rendite insgesamt und für ihre einzelnen Kanäle so gut wie möglich geprüft werden sollte. Katz et al. (1974) haben postuliert, dass Menschen Medien vor allem nach dem subjektiven Nutzen aussuchen, den sie durch deren Rezeption erfahren. Sie betonen, dass die Einlösung erwarteten Nutzens von den Rezipienten als Belohnung empfunden wird. Dieser Effekt präge das Verhalten der Menschen stärker als die vom Absender intendierte Wirkung der Medien. Die Mitarbeiter und Partner stellen sich in jedem Fall die Frage, wie viel Aufwand ihre Organisation treibt, um sie physisch zu erreichen, um Fakten verständlich zu machen und sie schließlich emotional wie kognitiv zu überzeugen. Was sie über diesen Aufwand denken, teilen sie nicht immer den Urhebern der Kommunikation selbst mit, sondern diskutieren sie viel eher in der Kantine.

Wechseln die Botschaften zu häufig werden sie weder beachtet noch wirken Glaubwürdigkeit muss über die Zeit erarbeitet werden. Der gesunde Menschenverstand - und die Einsicht in die eigenen Empfindlichkeiten getreu Kants kategorischem Imperativ - bewahrt auch die offiziellen Kommunikatoren vor den gröbsten Fehlern.

\subsection{Zusammenfassung}

Professionelle Organisationen zeichnen sich dadurch aus, dass in diesen eine Gruppe von Menschen gemeinsam an einem Ziel oder einer Aufgabe arbeiten. Interne Kommunikation bezeichnet den organisierten Informationsaustausch zwischen den Angehörigen einer solchen Organisation, um diese Ziele zu erreichen. Die gesteuerte Kommunikation wird dabei automatisch von der spezifischen Gruppendynamik der Beteiligten überlagert. Es lohnt sich daher, auf den dokumentierten Erfahrungen von Fachgemeinschaften aufzubauen, die sich gut auf die Strukturen wissenschaftlicher Forschungsverbünde übertragen lassen.

Die Arbeit in großen Forschungsverbünden ist gelebte Kommunikation. Wenige, simple Regeln helfen bei deren Gestaltung: Das Team gut starten. Teamcharta abfassen. Gruppenprozesse kennen und berücksichtigen. Vertrauen untereinander fördern. Das Team zusammenhalten. Strukturiert kommunizieren.

Eine systematische Medienwahl basiert darauf, die Medien-Zugangsmöglichkeiten der Teammitglieder und die Eigenschaften der Medien zu kennen und zu berücksichtigen; eine einfache Basisstruktur der Kommunikation festzulegen, eine passende Sprache zu finden; die Kultur der Organisation zu reflektieren und Inhalte vom Empfänger her zu planen.

Institutionelle Kommunikation wird von den Mitarbeitern danach beurteilt, wie umfassend sie individuell wahrgenommene Fragen aufgreift und beant- 
wortet. Tut sie dies nicht, wird sie ignoriert und durch individuelle Kommunikation ersetzt. Je weiter die Entfernung der Menschen voneinander ist und je geringer ihr Vertrauen in die organisatorischen Strukturen, desto wählerischer werden sie - bei den Medien, wem sie zuhören, was sie glauben und wem sie selbst die Wahrheit sagen. Die Konsistenz aller von den Menschen erlebten Maßnahmen entscheidet dabei mehr über den Erfolg der internen Kommunikation als der angebotene Medienmix.

\section{Quellenangaben}

Asch S.E. (1946): Forming Impressions of Personality. Journal of Abnormal and Social Psychology 41: 258-290

Buckingham M., Coffmann C. (2002): Erfolgreiche Führung gegen alle Regeln. Frankfurt/New York

Diehl M., Ziegler R. (2000): Informationsaustausch und Ideensammlung in Gruppen. In: Boos M., Jonas K.J., Sassenberg K. (Hrsg.): Computervermittelte Kommunikation in Organisationen. Hofgrefe, Göttingen Doppler K. (2007): Führen in Zeiten der Veränderung. In: Organisationsentwicklung, 1/06: 28ff., Düsseldorf Hein F.M. (2006): Führen im Netz. In: Handbuch PersonalEntwickeln, 108,12/2006, Köln

Hein F.M. (2007): Elektronische Unternehmenskommunikation - Konzepte und Best Practices zu Kultur und Führung. Frankfurt

Hildreth P.M., Kimble C., Wright P. (1998): Computer mediated communications and international communities of practice. In: Proceedings of Ethicomp ' 98 . Rotterdam, S. 275-286

Hildreth P.M., Kimble C.; Wright P. (2000): Communities of Practice in the distributed international environment. In: Journal of Knowledge Management 4(1): 27-38

Janis W. (1973): Victims of Groupthink: A Psychological Study of Foreign Policy Decisions and Fiascoes. Boston

Katz E., Blumler I., Gurevich M. (1974): Utilization of Mass Communication by the Individual. In: The Uses of Mass Communication. Current Perspectives on Gratification Research. Beverly Hills

Kim A.J. (1999): Conversation with fullcirc.com. 0.0.

Kim A.J. (2000): Community Building on the web: Secret strategies for successful online communities. Berkeley Krishna S., Sahay S., Walsham G. (2004): Managing cross-cultural issues in Global software outsourcing. In: Communications of the ACM, H. 4, Washington

Lipnack I. (1995): The Organisation of Future Solutions. 0.0.

Lipnack J. (2000): Virtual Teams: The Future is now. Linezine. Lipsack

Majchrzak A., Malhotra A., Stamps I., Lipnack J. (2004): Can absence make a team grow stronger. In: Harvard Business Review, H. 4, Cambridge

Morello D., Burton B. (2006): Future Worker 2015: Extreme Individualization. In: Gartner Research, 3/06, Stamford

Sackmann S.A. (2006): Betriebsvergleich Unternehmenskultur. Welche kulturellen Faktoren beeinflussen den Unternehmenserfolg? Universität der Bundeswehr. München

Schein E.H. (2004): Organizational Culture and Leadership. San Francisco

Surowiecki J. (2004): The Wisdom of Crowds. 0.0.

Wainfan L., Davis P.K. (2004): Challenges in virtual Collaboration. Videoconferencing, Audioconferencing, and Computer-Mediated Communications. Rand Corporation

Wenger E. (1998): Communities of Practice: Learning, Meaning and Identity. Cambridge

Wenger E., McDermott R.M., Snyder W.M. (2002): Cultivating Communities of Practice. Harvard

Wertekommission (2007): Führungskräftebefragung. Online verfügbar unter: http://www.wertekommission. de/content/pdf/kampagne/wk_studie.pdf

Wink R. (2007): Elektronische Kommunikation in der Wissenschaft: Fallbeispiel Stammzellforschung. In: Hein, Frank Martin (2007): Elektronische Unternehmenskommunikation - Konzepte und Best Practices zu Kultur und Führung. 


\section{Weiterführende Literatur}

Brock D.W. (2006): Is a consensus possible on stem cell research? Moral and political obstacles. Journal of Medical Ethics 32(1): 36-42.

Camargo A., Winterhager M. (2006). Internationale Kooperation in der Stammzellforschung. Eine bibliometrische Analyse am Beispiel der Länder Deutschland, Kanada, Niederlande, Schweden, USA und Vereinigtes Königreich. In: Deutsche Stammzellpolitik im Zeitalter der Transnationalisierung, hrsg. v. R. Wink, BadenBaden; Nomos, 45-74.

Cooke P. (2005): Rational drug design, the knowledge value chain, and bioscience megacentres. Cambridge Journal of Economics, 29, 325-341.

Pompe S., Bader M., Tannert C. (2005): Stem-cell research: the state of the art. European Molecular Biology Organization reports, 6, 297-300.

Powell W.W., White D.R. et al. (2005): Network dynamics and evolution. The growth of interorganizational collaboration in the life sciences. American Journal of Sociology 110(4): 1132-1205.

Wink R. (2006): Transnationalisierung und Standortwettbewerb in der Stammzellforschung. In: Deutsche Stammzellpolitik im Zeitalter der Transnationalisierung, hrsg. v. R. Wink, Baden-Baden; Nomos, 177-195. 